\title{
Comparative Study of Using Vein Finder and Common Venipuncture Method in Anxiety and Psychological Indicators of 3-6-years Old in the Ward of Pediatric Internal
}

\author{
Zahra Alizadeh ${ }^{1 *}$ (D) \\ ${ }^{1}$ MSc, Department of Pediatric Nursing, School of Nursing and Midwifery, Shiraz University of Medical Sciences, Shiraz, Iran \\ * Corresponding author: Zahra Alizadeh E-mail: z.alizade8871@yahoo.com ORCID: 0000-0002-0660-3297 \\ Received: 25 March 2021 Accepted: 17 August 2021
}

\begin{abstract}
Introduction \& Background: The current study aimed to compare the effect of vein finder device and conventional venipuncture technique on anxiety and physiological indices of children with 3-6 years old in pediatric medical care.

Methods and Materials: This is a randomized clinical trial which was conducted on 62 children between 3-6 Years old who were eligible according to the study inclusion criteria. This study was patients hospitalized in pediatric ward of Namazi hospital, Shiraz-IRAN, 2015. Vein finder device was applied in the intervention group while the control group receives the conventional venipuncture technique. Anxiety responses such as heart rate, respiratory rate, blood pressure, and blood oxygen saturation were assessed at 5 minutes both before and after the intravenous catheter placement procedure in both groups. Yale Preoperative Anxiety Scale (YPAS) was used to measure children's anxiety scale. The data were analyzed by using paired t-test, independent t-test, and ANOVA test in SPSS software.

Results: Both groups were measured after the intervention. Parametric physiologic results reveal a significant difference in heart rate $(p<0.001)$ and respiratory rate ( $p: 0.002)$ for both groups. No significant difference was reported for blood pressure and blood oxygen saturation. Mean anxiety score was reported 13/77 $\pm 1 / 94$ in the intervention group and 18 $2 / 44$ in the control group after applying the intervention ( $p: 0.001$ ).
\end{abstract}

Conclusion: Study findings indicate a significant decrease of anxiety score for intervention group after the IV insertion. vein finder technology device was along with positive effects on anxiety reduction and physiologic indices balance for children between 3-6 years old, it can be applied as a proper method for IV catheterization in pediatric medical wards.

Keywords: anxiety, IV catheterization, hospitalized children

\section{INTRODUCTION}

According to Wong, illness and hospitalization are the first crisis children encounter During early years of life [1].
Hospital care and invasive or frightening treatment experiences have important psychological impacts on children [2]. Invasive procedures can be incredibly stressful 
and devastating for pediatric patients [3]. Many pre-school children find almost everything in an unusual, but older children concern about their independence and freedom [4]. Hospitalized children and those who come for outpatient appointments experience miserable and painful procedures including IV catheter insertion, blood sampling, biopsy, etc. [5]. Venipuncture is a procedure through that a plastic tube is inserted into the venous system. This procedure commonly used in diagnosing and treatment. Moreover, it's the fastest way to administer fluids, electrolytes, nutrients, antibiotics, blood, and drug into the central or peripheral venous circulatory system [6]. In fact, it is one of the oldest most common and painful invasive procedures in nursing [7]. In another word, about $90 \%$ of patients may require this invasive procedure [8].

About 40 percent of all medicines and fluids are used through an IV line [9]. The results of a survey showed that 74 percent of children announced this procedure as their worst miserable experience [10]. Physiologic changes during anxiety are including fast breathing, fast pulse, heavy sweating, red and skin, high blood pressure, dilated pupils, and appetite loss [11]. IV catheterization requires a high level of expertise and accuracy in patients, especially children, due to their generalized edema, body soft tissue, and the condition of their veins (complicated vascular access) [12]. Sometimes our first attempt is not along with success, so you're bound to have a few failures [13].

Recently, vein detector technology device can aid and facilitate this process. This device is used to indicate the vessel. The primary reason is the required sensitivity and accuracy in carrying out intravenous injection and blood sampling. Moreover, the features like: first-attempt vascular access success rates were significantly higher, the number of attempts per patient was significantly lower, detecting the most proper vein within 5 seconds, the ability to distinguish vein from the artery and its high speed make it more popular [14]. Its appliance leads to decrease in the number of staff attempts, more time and money saving, higher staff speed and efficiency, and more patients safety, satisfaction and comfort. Its usage was associated with a reported success rate of $100 \%$ and less time spending in vein detecting for $25 \%$ [15]. The importance of reducing children's anxiety during catheter insertion and its consequences, despite its high efficiency, leads us to design this survey. This study aims to compare children's anxiety by vein finder technology device and conventional venipuncture technique.

\section{METHOD AND MATERIAL}

This is a randomized clinical trial which was conducted on 62 children aged 3-6 years who were hospitalized in pediatric medical wards of Nemazi hospital, Shiraz-IRAN, 2015. They were randomly allocated into Intervention group $(n=31)$ and Control group $(n=31)$. We select this center in terms of its more and diverse pediatric units, more specialties and the large number of patients who refer to this center.

The study inclusion criteria including parental consent for children to participate in the study, children's age range from 6-3 year's old, confirmed stability in physiological parameters by a physician, and hydration order by a physician.

Exclusion criteria including parents' reluctance to continue his company in the research or their company cut off due to any reason during the study, and finally child's death.

Envelope distribution method was considered for random allocation technique. We wrote the names of both intervention and control groups on the beginning of sampling. Then, the envelopes were opened randomly for each patient. We follow this to the end of sampling and patients were accidentally allocated into each of two groups.

To increase the accuracy and homogenization of the project, we plan to ask a fixed person for intravenous catheterization in the treatment room.

Data collection was done by applying Demographic information questionnaire; which consists of two parts related to the child, including age, gender, diagnosis, location, the frequency, and length of hospitalization and in the next part related to the parents, including age and parents education. Data were collected by interviewing parents and recording file information. Child physiologic indices such as heart rate, respiratory rate, blood pressure, and blood oxygen saturation were assessed at 5 minutes both before and after the intravenous catheter placement procedure in both groups. YALE PAS was used to measure children's anxiety scale. Children's anxiety responses were registered through recording the pictures and videos at tensecond intervals. YALE PAS scale contains five categories (activity, emotional expressivity, state of arousal, vocalization, and use of parents).

In two other studies, this method was used in America and Tehran respectively. This technique was administered to 51 children ( 27 male and 24 female patients) within 3 phases in 
ZeevKain survey including Pre-catheterization phase (T1) and post-catheterization phase $(\mathrm{t} 2)[16,17]$.

Its validity as assessed by the correlation between the Yale Preoperative Anxiety Scale (YPAS) total score and the STAIC was good $(P=0.01, Y=0.79)$. Construct validity was assessed by comparing the Yale Preoperative Anxiety Scale (YPAS) scores at three increasingly stressful points.

There were significant differences in Yale Preoperative Anxiety Scale (YPAS) scores over the three stress points ( $F$ $[1.36]=0.6, P=0.001)$.

Questionnaire reliability was assessed by applying k-factor which revealed the agreement between observers ranged from 0.68 to 0.86 . Construct validity was $p: 0.001$ [18]. This study supports the validity of the anxiety scale. Visible children anxiety symptoms were recorded by this scale. In this study, we applied an intra-expert to determine and boost the recorded data reliability by her evidence. Observers received some training prior to study but she was unaware of study hypotheses. After the intervention, videos were carefully checked by the researchers to measure the checklist items by means of anxiety scale. Finally, each category was scored. To increase the accuracy and homogenization of the project, we plan to ask a fixed person for intravenous catheterization in the treatment room. Mean heart rate and respiration, blood pressure and arterial blood oxygen saturation were measured for both groups as follow: we used a pulse oxymeter to measure heart rate, blood pressure, and blood oxygen saturation. Blood pressure was checked by pediatric sphygmomanometer with ageappropriate cuff (for children between 3-6 years old).

Then, to determine the child's anxiety based on YaLe- PASS scale, we used fixed camera recorder. This fixed camera had closed view shots for 5 minutes both before and after intravenous catheterization in both groups. Child's recorded file was reassessed by an intra-expert to determine and boost the recorded data reliability by her evidence. Observers received some training prior to study but she was unaware of study hypotheses. Observed changes (according to the checklists) were scored by means of anxiety scale. Anxiety Scores range from 5 to 22 with higher scores indicating greater anxiety. Finally, physiological indices (both after and before intravenous catheterization) were compared between both groups. The data were analyzed by using paired t-test, independent t-test, and ANOVA test in SPSS software.

\section{RESULTS}

The mean age of the children in the intervention group was $4.27 \pm 1.20$ years and in the control group $4.38 \pm 1.11$ years. Independent t-test showed no significant difference between the two groups in terms of age ( $p: 0.77)$. The majority of children in both intervention (74.2\%) and control group (71\%) were boy. A chi-square test shows that there is no significant difference between the two groups in terms of gender ( $p$ : $0.77 \%$ ). Parental education level was categorized into (elementary -undergraduate) and (Diploma-Bachelor and above). Parents education level (elementary -undergraduate) was (mother: 16.1, father: 32.3) in the intervention group and (mother: 64.5, father: $67.5)$ in the control group. on the other hand, DiplomaBachelor and above level were (mother: 83.09, father: 67.7) in the intervention group and (mother: 35.5 , father: 32.3 ) in the control group.

Chi-square test indicated a significant difference in the frequency of parents' education level between two groups [fathers ( $p: 0.005)$, mothers $(p<0.001)]$. Children hospitalization period was divided into two groups: 2-1 days and three and above. Chi-square results showed equal mean hospitalization duration for both the intervention and control groups which implies no significant difference $(p$ : 0.63). Hospitalization frequency was divided into two groups: two and below two days and above two days. Chisquare results showed no equal mean hospitalization frequency for both the intervention and control groups which implies a significant difference (p: 0.02). In the intervention group, about 24 people had the most number of hospitalizations along with less than 2 days hospitalization (77.4\%) and just 7 people had the lowest number of hospitalizations along with more than 2 days hospitalization (22.6\%). minutes before venipuncture in both intervention and control groups (Tables 1-5).

Independent t-test shows a lower heart rate for the control group (in compared with before the intervention) $(p<0.001)$. Paired t-test was applied to both groups separately, which reveals a significant difference between both groups in compared to before intervention (p:0.006). ANCOVA analysis found a significant difference between two groups after the intervention while removing the effect of the primary heart rate $(p<0.001)$.

Independent t-test reveals not significant difference in terms of respiratory rate, so their respiratory rate was matched before the intervention ( $p: 0.9$ ). Comparing the post-pre intervention in each group (by paired t-test) showed a significant difference in terms of the mean 
Table 1. The average of changes in Heart Rate five minutes after the venipuncture in compare to five minutes before venipuncture in both intervention and control groups

\begin{tabular}{c|c|c|c|}
\hline \multirow{2}{*}{ Test results } & $\begin{array}{c}\text { Control } \\
\text { Conventional venipuncture }\end{array}$ & $\begin{array}{c}\text { Intervention } \\
\text { Vein finder device }\end{array}$ & \multirow{2}{*}{$\begin{array}{c}\text { Group } \\
\text { heart rate }\end{array}$} \\
\cline { 2 - 4 } & $(\mathrm{M} \pm \mathrm{SD})$ & $(\mathrm{M} \pm \mathrm{SD})$ & minutes before venipuncture \\
\hline${ }^{*} \mathrm{t}=7.3, \mathrm{df}=60, \mathrm{p}<0.001$ & $04.10 \pm 105$ & $6.11 \pm 115$ & after 5 minutes venipuncture \\
\hline $\mathrm{t}=-0.81, \mathrm{df}=60, \mathrm{P}=0.42$ & $6.8 \pm 113$ & $39.10 \pm 112$ & ${ }^{\star \star *}$ test results \\
\hline${ }^{* *} \mathrm{~F}=21.15, \mathrm{df}=1, \mathrm{p}<0.001$ & $\mathrm{t}=5.720, \mathrm{df}=30, \mathrm{p}<0.001$ & $\mathrm{t}=2.97, \mathrm{df}=30, \mathrm{P}=0.006$ & \\
\hline
\end{tabular}

Table 2. The average of changes in respiratory rate five minutes after the venipuncture in compare to five minutes before venipuncture in both intervention and control groups

\begin{tabular}{c|c|c|c|}
\hline \multirow{2}{*}{ Test results } & $\begin{array}{c}\text { Control } \\
\text { Conventional venipuncture }\end{array}$ & $\begin{array}{c}\text { Intervention } \\
\text { Vein finder device }\end{array}$ & $\begin{array}{c}\text { Group } \\
\text { respiratory rate }\end{array}$ \\
\cline { 2 - 4 } & $(\mathrm{M} \pm \mathrm{SD})$ & $(\mathrm{M} \pm \mathrm{SD})$ & 5 minutes before venipuncture \\
\hline${ }^{*} \mathrm{t}=-0.1, \mathrm{df}=60, \mathrm{P}=0.9$ & $04.10 \pm 105$ & $2.63 \pm 26$ & after 5 minutes venipuncture \\
\hline $\mathrm{t}=-3.16, \mathrm{df}=60, \mathrm{P}=0.002$ & $5.65 \pm 28.4$ & $3.01 \pm 24.67$ & ${ }^{* * *}$ test results \\
\hline${ }^{* *} \mathrm{~F}=36.91, \mathrm{df}=1, \mathrm{p}<0.001$ & $\mathrm{t}=-4.29 \mathrm{df}=60, \mathrm{p}<0.001$ & $\mathrm{t}=5.88, \mathrm{df}=30, \mathrm{p}<0.001$ & \\
\hline
\end{tabular}

Table 3. The average of changes in blood pressure Systol five minutes after the venipuncture in compare to five minutes before venipuncture in both intervention and control groups

\begin{tabular}{|c|c|c|c|}
\hline \multirow{2}{*}{ Test results } & $\begin{array}{c}\text { Control } \\
\text { Conventional venipuncture }\end{array}$ & $\begin{array}{c}\text { Intervention } \\
\text { Vein finder device }\end{array}$ & \multirow{2}{*}{$\begin{array}{c}\text { Group } \\
\text { blood pressure Systol }\end{array}$} \\
\cline { 2 - 4 } & $(\mathrm{M} \pm \mathrm{SD})$ & $(\mathrm{M} \pm \mathrm{SD})$ & 5 minutes before venipuncture \\
\hline${ }^{*} \mathrm{t}=-0.1, \mathrm{df}=60, \mathrm{P}=0.73$ & $9.50 \pm 100$ & $18 \pm 99$ & after 5 minutes venipuncture \\
\hline $\mathrm{t}=10.5, \mathrm{df}=60, \mathrm{P}=0.025$ & $8.22 \pm 99.67$ & $16.98 \pm 103$ & ${ }^{\star * *}$ test results \\
\hline $\mathrm{F}=2.250^{* *}, \mathrm{df}=60, \mathrm{P}=0.13$ & $\mathrm{t}=-1.6, \mathrm{df}=60, \mathrm{P}=0.25$ & $\mathrm{t}=-1.54, \mathrm{df}=60, \mathrm{P}=0.13$ & \\
\hline
\end{tabular}

Table 4. The average of changes in blood pressure Diastol five minutes after the venipuncture in compare to five minutes before venipuncture in both intervention and control groups

\begin{tabular}{c|c|c|c|}
\hline \multirow{2}{*}{ Test results } & $\begin{array}{c}\text { Control } \\
\text { Conventional venipuncture }\end{array}$ & $\begin{array}{c}\text { Intervention } \\
\text { Vein finder device }\end{array}$ & \multirow{2}{*}{$\begin{array}{c}\text { Group } \\
\text { blood pressure Diastol }\end{array}$} \\
\cline { 2 - 4 } & $(\mathrm{M} \pm \mathrm{SD})$ & $(\mathrm{M} \pm \mathrm{SD})$ & 5 minutes before venipuncture \\
\hline${ }^{*} \mathrm{t}=1.24, \mathrm{df}=60, \mathrm{P}=0.21$ & $10.45 \pm 72.4$ & $11.87 \pm 74$ & after 5 minutes venipuncture \\
\hline $\mathrm{t}=1.24, \mathrm{df}=60, \mathrm{P}=0.21$ & $11.32 \pm 73.6$ & $12.14 \pm 76$ & ${ }^{* * *}$ test results \\
\hline $\mathrm{f}=1.19^{* *}, \mathrm{df}=1, \mathrm{P}=0.27$ & $\mathrm{t}=0.21, \mathrm{df}=60, \mathrm{P}=0.83$ & $\mathrm{t}=0.3, \mathrm{df}=60, \mathrm{P}=0.76$ & \\
\hline
\end{tabular}

Table 5. The average of changes in blood oxygen saturation five minutes after the venipuncture in compare to five minutes before venipuncture in both intervention and control groups

\begin{tabular}{c|c|c|c|}
\hline \multirow{2}{*}{ Test results } & $\begin{array}{c}\text { Control } \\
\text { Conventional venipuncture }\end{array}$ & $\begin{array}{c}\text { Intervention } \\
\text { Vein finder device }\end{array}$ & \multirow{2}{*}{$\begin{array}{c}\text { Group } \\
\text { blood oxygen saturation }\end{array}$} \\
\cline { 2 - 4 } & $(\mathrm{M} \pm \mathrm{SD})$ & $(\mathrm{M} \pm \mathrm{SD})$ & 5 minutes before venipuncture \\
\hline${ }^{*} \mathrm{t}=-1.43, \mathrm{df}=60, \mathrm{P}=.015$ & $2.91 \pm 99.6$ & $3.26 \pm 19.96$ & after 5 minutes venipuncture \\
\hline $\mathrm{t}=-1 / 34, \mathrm{df}=60, \mathrm{p}=0 / 18$ & $16.35 \pm 6 / 92$ & $2.91 \pm 96.6$ & ${ }^{\star * *}$ test results \\
\hline${ }^{* *} \mathrm{~F}=2.50, \mathrm{df}=1, \mathrm{P}=0.11$ & $\mathrm{t}=1.5, \mathrm{df}=60, \mathrm{p}=0.13$ & $\mathrm{t}=-1.53, \mathrm{df}=60, \mathrm{p}=0.13$ & \\
\hline
\end{tabular}

respiratory rate $(p<0.001)$. ANCOVA analysis found a significant difference between two groups after the intervention while removing the effect of the primary respiratory rate $(p<0.001)$.

Independent t-test reveals not a significant difference in terms of systolic blood pressure, so their systolic blood pressure was matched before the intervention (p: $0.73, d$ : -
1). Comparing the post-pre intervention in each group (by paired t-test) showed no significant difference in terms of the systolic blood pressure ( $p: 0.13$ ). ANCOVA analysis found a significant difference between two groups after the intervention while removing the effect of the systolic blood pressure $(p<0.001)$. 
Table 6. The average anxiety score five minutes after the venipuncture in compare to five minutes before venipuncture in both intervention and control groups

\begin{tabular}{c|c|c|c|}
\hline \multirow{2}{*}{ Test results } & $\begin{array}{c}\text { control } \\
\text { Conventional venipuncture }\end{array}$ & $\begin{array}{c}\text { intervention } \\
\text { Vein finder device }\end{array}$ & \multirow{2}{*}{$\begin{array}{c}\text { group } \\
\text { anxiety }\end{array}$} \\
\cline { 2 - 4 } & $(\mathrm{M} \pm \mathrm{SD})$ & $(\mathrm{M} \pm \mathrm{SD})$ & 5 minutes before venipuncture \\
\hline $\mathrm{t}=-7.52, \mathrm{df}=60, \mathrm{p}<0.001$ & $2.04 \pm 19.38$ & $2.18 \pm 17.48$ & 5 minutes after venipuncture \\
\hline $\mathrm{t}=-7.52, \mathrm{df}=60, \mathrm{p}<0.001$ & $2.44 \pm 18$ & $\mathrm{t}=10.49 \pm 13.77$ & test results ${ }^{* * *}$ \\
\hline $\mathrm{F}=36.98^{* *}, \mathrm{df}=1, \mathrm{p}<0.001$ & $\mathrm{t}=4, \mathrm{df}=30, \mathrm{p}<0.001$ & $\mathrm{p}<0.001$ & \\
\hline
\end{tabular}

Independent t-test reveals not a significant difference in terms of diastolic blood pressure, so their diastolic blood pressure was matched before the intervention ( $p$ : 0.73). Comparing the post-pre intervention in each group (by paired t-test) showed no significant difference in terms of the diastolic blood pressure ( $p: 0.76)$. ANCOVA analysis found no significant difference between two groups after the intervention while removing the effect of the primary diastolic blood pressure (p: 0.27).

Independent t-test reveals not a significant difference in terms of blood oxygen saturation, so their blood oxygen saturation was matched before the intervention ( $p: 0.15)$. Comparing the post-pre intervention in each group (by paired t-test) showed no significant difference in terms of the blood oxygen saturation ( $p: 0.13$ ). ANCOVA analysis found no significant difference between two groups after the intervention while removing the effect of the primary blood oxygen saturation (p: 0.11).

There was a decrease in the mean blood oxygen saturation after venipuncture in the group applied vein finder (d: 4) (Table 6).

Independent t-test reveals a significant difference in terms of anxiety, so their primary anxiety was not matched before the intervention $(p<0.006)$. Comparing the post-pre intervention in each group (by paired t-test) showed a significant difference in terms of the anxiety $(p<0.001)$. ANCOVA analysis found a significant difference after the intervention ( $p: 0.001$ ). There was a decrease in anxiety level after venipuncture in the vein finder group.

\section{DISCUSSION}

According to the results, vein finder technology device keeps the child heart rate stable. It helps to prevent heart rate alteration at venipuncture, so it's along with less heart beat changes in children. According to researcher study, there is no evidence of documented survey about the impact of vein finder technology device on the anxiety of children within 3-6 years old. This study finding is consistent with other previous results which were done about the effect of venipuncture on heart rate. According to study was conducted by Kalani (2004) in Shiraz to examine the effect of EMLA cream on physiologic criteria such as heart and respiratory rate. The results revealed more balanced physiological indices after intravenous catheterization for children in the intervention group [19]. According to these findings, vein finder technology device can be along with more stable heart rate in children, so it can be introduced as the proper facilitator in IV insertion. The results showed that, no significant difference was found in terms of the mean respiratory rate before catheter insertion in both groups. But there was a statistically significant rise in the average number of respiration rate after venipuncture in the control group than in the intervention group. Increased respiratory rate is another signs of anxiety response in children. Its occurrence along with conventional method of IV insertion implies its stressful impact. The results showed that $r$ a significant difference in terms of the mean respiratory rate before and after intervention for both groups.

After venipuncture, the number of breaths was increased in the control group while it was decreased in the intervention group. Since the respiratory rate is one of the anxiety elements, this decrease can be rooted in the mitigating effect of vein finder technology device on children anxiety.

Previous studies about IV insertion effect on blood pressure were inconsistent due to the lack of significant difference. Fixler conducted a study about conventional IV insertion effect on pediatric systolic blood pressure in 1980. Data analysis showed an increase for the intervention group, but it was not significant statistically [20]. In this study, systolic blood pressure had lower changes. According to the results showed that, the systolic blood pressure showed no significant difference before venipuncture in both groups ( $p$ : 0.73). After IV insertion, mean diastolic blood pressure was higher in the intervention group, but it was not significant statistically. Children's reduction of diastolic blood pressure in conventional venipuncture technique may root in its dreadful concept among children in compare to the vein finder device appliance. Comparing the post-pre intervention in each group showed no significant difference in terms of the diastolic blood pressure. The results showed that no significant difference in terms of diastolic blood 
pressure in both groups after the intervention. Comparison of diastolic blood pressure changes at before and after venipuncture showed a non-significant increase in both control and intervention groups. There is no evidence of documented survey about the impact of vein finder technology device on the diastolic blood pressure. According to Sudung study (2003), vein puncture procedure caused significant alteration and rise in diastolic blood pressure [21]. The results of this study revealed a less diastolic blood pressure alteration among children The results of this study were inconsistent with those had been done about conventional IV insertion effect on diastolic blood pressure for its non-significant differences The results showed that no significant difference in terms of mean blood oxygen saturation before the intervention in both groups. Comparing the post-pre intervention in each group showed no significant difference in terms of the blood oxygen saturation. The results showed that no significant difference between two groups after the intervention. In the control group, we had lower mean blood oxygen saturation (after the IV insertion) in compared to the intervention group. Since low blood oxygen saturation anticipates the anxiety level in children, this decrease can contribute to the miserable effect of conventional IV insertion in compare to the vein finder device. Vosoghi did study about the effects of distraction on blood oxygen saturation in children aged 3-6 undergoing IV injection in 2010. Results showed a significant difference for blood oxygen saturation in the intervention group in comparison with the control group after the intervention $(p<0.001)$ [22]. Before the intervention, the findings did show a significant difference in mean anxiety score in two groups, so it implied a statistically significant difference in anxiety severity in two groups and methods. The results showed that a significant difference in mean anxiety score at 5 minutes after the intravenous catheter placement procedure in both groups. Therefore, there is a statistically significant difference after the intervention $(p<0.001)$. Obviously, IV insertion is a miserable procedure for all children, so they experience a level of anxiety before the procedure regardless to the techniques applied. According to this study, the intervention group had a lower level of anxiety after the IV insertion. A paired t-test was applied to compare the anxiety score before and after the intravenous catheter placement procedure in both groups, which showed a significant difference [23].

\section{CONCLUSION}

A significant difference was found in the mean anxiety score of the intervention and control groups which states a lower stress for the intervention group than the latter one. Uman and his colleague did study to examine the impact of IV insertion with the conventional method on children's distress in 2006 [24]. It showed that this procedure was associated with anxiety responses such as frown face, crying and restlessness in children. Therefore, it can be applied as a proper method for IV catheterization in pediatric medical wards. According to the findings and conclusions in this research can be concluded that. Since vein finder device shows a positive impact on physiologic responses of anxiety in children during IV insertion, it can be suggested as the proper, safe, effective, low-cost and tension procedure for preschool and high-risk children. Furthermore, it's along with more patient, parents and nurses satisfaction which lead to more quality of nursing care and develops research scope in this regard.

Author contributions: All authors were involved in concept, design, collection of data, interpretation, writing, and critically revising the article. All authors approve final version of the article.

Funding: The authors received no financial support for the research and/or authorship of this article.

Acknowledgements: The authors acknowledge the responsible, respectful staff and nurses of Namazi Hospital in Shiraz.

Declaration of interest: Authors declare no competing interest.

Data availability: Data generated or analysed during this study are available from the authors on request.

\section{REFERENCES}

1. Wong $D$, Hockenberry $M$, Wilson $D$, Winkelstein $M$, Kline Ne. Wong's Nursing care of infants and children $\left(7^{\text {th }}\right.$ louis ed.) Mosbey, 2019; 26(5): 159-75.

2. Maclaren J, Cohen L. A comparison of distraction strategies for venipuncture distress in children. Journal of Pediatric Psychology, 2017; 30(5): 387-96. (doi: 10.1093/jpepsy/jsi062).

3. Hines S. Children's hospital awareness initiative: Improving employee communications with pediatrics. Poster presented at Lvhn Research Scholar Program Poster Session, Lehigh Valley Health Network, Allentown. 2018; 15(2): 156-70.

4. Bowden V. Greenberg CS. Pediatric Nursing Procedures, Philadelphia Lippincott, 2020; 17(4): 156-78.

5. Hands C, Round J, Thomas J. Evaluating venepuncture practice on a general children's ward. Paediater Nurse, 2019; 22(5): 32-5. (doi: 10.7748/paed2010.03.22.2.32.c7597).

6. Infusion Nurses Society. Infusion nursing standards of practice. Journal of Infusion Nursing, 2006; 29(1 Suppl): S1-92. (doi: 10.1097/00129804-200601001-00001). 
7. Burr S. Pain in childhood. Journal of Nursing, 2019; 24(1): 1-4.

8. Taghinejad $\mathrm{H}$, Asadizaker $M$. Radiation comparative study of the effects of alcohol and iodine as a disinfectant Brvarz Catheter insertion site of catheters. Journal of Ilam University of Medical Sciences, 2016; 13(4): 10-6.

9. Tayyebi S, Shiva N. The comparison of the two methods Phlebitis dressing with and without the use of topical antiseptics venous catheter location. Journal - College of Nursing and Midwifery Mashhad Fifth Year Spring and Summer XVI No, 2016; 15(5): 33-45.

10. Razavi M. Evaluation of the risk of superficial thrombophlebitis of application of intravascular devices and its risk factors in patients admitted to hospital in the law. First National Congress of Public Health and Preventive Medicine, 2016; 8(3): 1-3.

11. Dougherty L, Lamb J. Peripheral cannulation. Nurse, 2017; 22(5): 49-56. (doi: 10.7748/ns2008.09.22.52.49.c6650).

12. Azizi A. Improving peripheral IV cannula care: Implementing high-impact. Br J Nurse, 2018; 18(20): 1242-9. (doi: 10.12968/bjon.2009.18.20.45116).

13. Hoffman $H$, Kessler $H$, Eppel T, Rukavina $S$, Traue $H$. Expression intensity, gender and emotional recognition: Women recognize only subtle facial emotions better than men. Acta Psychologica, 2019; 135(3): 278-83. (doi: 10.1016/j.actpsy.2010.07.012).

14. Powell J, Tarnow K. The relationship between peripherial intravenouse catheter indwell time and incidence of phlebitis. Journal of Infusion Nursing, 2017; 31(1): 39-45. (doi: 10.1097/01.NAN.0000308544.67744.50).

15. Hess H. Biomedical device to improvement pediatric vein access success. Pediater Nurs, 2018; (36): 259-69.

16. Kain Z, Mayes L, Caramico L, Domenic V, Cicchetti D. Measurement tool for pre- oprative anxiety in children. The Preoperative Anxiety Scale. Anesth Analage; 1997; (85): 783-8.
17. Mehdizadeh $M$, Nakhaei Moghaddam M, Kolahi AA, Zamani Gh. Comparing the effects of IV and intranasal midazolam administration on pain, anxiety and fear of children undergoing painful invasive procedures. Pajoohandeh Journal; 2008; 13(2): 121-7.

18. Mehdizadeh M, Nakhaei Moghaddam M, Kolahi A, Zamani G. Comparing the effects of IV and intranasal midazolam administration on pain, anxiety and fear of children undergoing painful invasive procedures. Pajoohandeh Journal, 2015; 13(2): 121-7.

19. Kalani Tehrani D. Comparison of EMLA topical anesthetic cream and music on pain intensity caused by catheter insertion, Msc Dissertation. Shahid Beheshti University of Medical Sciences, 2017; 7(4): 130-48.

20. Fixler D, Kautz A, Dana K. Systolic blood pressure differences among pediatric, epidemiological studies, 2016; 13(2): 89-111.

21. Sudung P, Partini T, Taralan T. The influence of stressor on blood pressure in school children. Paediatrica Indonesiana, 2020; 43(3): 125-39.

22. Vosoghi N, Chehrzad M, Abotalebi G. Effects of distraction on physiologic indices and pain intensity in children aged 3-6 undergoing IV injection. Journal of Faculty of Nursing and Midwifery, 2017; 16(9): 307-14.

23. Uman L, Chambers C, Mcgrath P, Kisely S. Psychological interventions for needle-related procedural pain and distress in children and adolescents. Cochrane Database Syst Rev, 2019; 17(4): 179-95.

24. Uman LS, Chambers CT, Mcgrath PJ, Kisely S. Psychological interventions for needle-related procedural pain and distress in children and adolescents. Cochrane Database Syst Rev, 2006; (4): 179-95. (doi: 10.1002/14651858.CD005179.pub2). 